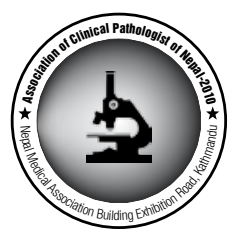

\title{
A systematic approach to cytological evaluation of central nervous system tumours
}

\author{
Panth $\mathrm{R}^{1}$ \\ ${ }^{I}$ National Institute of Neurological and Allied Sciences, Bansbari, Kathmandu, Nepal
}

\section{Keywords:}

Intraoperative consultation; Smear cytology;

Central nervous system neoplasm

\begin{abstract}
Smear cytology has been accepted as a suitable alternative to frozen section in intraoperative consultation of central nervous system tumours. Intraoperative smears from neurosurgical specimens permit rapid and accurate diagnosis. Grading of primary tumours may not be always possible on intraoperative smears, but their division into low or high-grade lesions is highly reliable and reproducible if due attention is paid to certain parameters like nuclear atypia, mitotic figures and necrosis. A systematic approach leads to a workable preliminary diagnosis, assures adequate tissue sampling for histological processing and guides the management team to explain the prognosis to the patient party and plan optimal treatment even before the final histological diagnosis is available.
\end{abstract}

\section{INTRODUCTION}

Brain tumours account for $2 \%$ of all cancers and $2.4 \%$ of all cancer deaths in USA. ${ }^{1}$ Tumours of the nervous system are the second most common childhood tumor after leukemia, constituting approximately $35 \%$ of all childhood malignancies and remain the leading cause of cancerrelated deaths in children. ${ }^{2,3}$ We do not have an organized brain tumour registry in Nepal, but a steady increase in the incidence of primary brain tumours over the last decade is logical because of higher detection rates due to more widespread availability of diagnostic imaging technique. As neurosurgery is not performed in many governmental and private hospitals in Nepal, it is only natural for a general

\section{Correspondence:}

\section{Dr Rajesh Panth, MD}

National Institute of Neurological and Allied Sciences

P.O. Box: 3711

Phone: 9851052766

Fax: 977-01-4370779

Email: rajeshreeta1981@yahoo.com pathologist to be a bit apprehensive when faced with the need to provide sensible interpretation of intraoperative neurosurgical specimens. We all need to be conversant and updated with a basic systematic approach so that we can build on it to devise our own personalized approach that best suits our management team.

\section{DISCUSSION}

\section{Smear cytology versus frozen section}

Smear cytology has been accepted as a suitable alternative to frozen section in intraoperative consultation of central nervous system (CNS) tumours. ${ }^{4}, 5$ Crisp morphological features provided by hematoxylin and eosin staining allows a rapid and accurate diagnosis by employing a technique that is simple, cheap and quick. Preservation of architecture is the major advantage of frozen section over smear cytology, while the major drawback is the common freezing artifacts that compromise proper evaluation. Unlike smear cytology, 
frozen section is more labour intensive and expensive and has limited application in Nepal because of lack of trained manpower.

In a large study comprising 4172 cases smears prepared from 3541 open biopsies and 631 stereotactic procedures, complete correlation with histopathology was achieved in $89.8 \%$ with interpretation being most accurate for meningiomas $(97.9 \%)$ followed by metastasis $(96.3 \%)$, oligodendroglioma (80.9\%) and ependymoma (77.7\%). ${ }^{6}$ Despite its worldwide familiarity and applicability, discrepant results were seen in 57 of the 2156 cases (2.7\%) analyzed by frozen section. Oligodendrogliomas could not be separated from astrocytomas in $21.1 \%$ of cases and gliosis could not be differentiated from gliomas in $14 \%$ of cases. Tumours were overgraded in $7 \%$ of cases. Spindle cell lesions and CNS lymphomas were the most difficult to decipher by frozen section with discrepant results occurring in $21.1 \%$ of cases each. ${ }^{7}$

\section{Application of smear cytology}

Intraoperative consultation with smear cytology is requested for after both closed stereotactic sampling procedure and open therapeutic surgical resections. A review of 7471 stereotactic biopsies revealed morbidity and mortality rates of $3.5 \%$ and $0.7 \%$ respectively while providing diagnosis in $91 \%$ of cases. ${ }^{8}$ The small bits of grayish white tissue provided by the stereotactic technique requires the pathologist to comment on the adequacy of the specimen so that representative tissue is assured for histological tissue processing. Preliminary diagnosis after open procedures guides the neurosurgeon during the open operative procedure and helps him to explain the likely prognosis to the apprehensive patient party immediately after the operation. ${ }^{9}$

\section{Challenges of cytological evaluation}

Crush smear cytology for CNS tumor diagnosis is challenging for the pathologist for many reasons. All biopsy specimens appear similar to shreds of white pulp in saline or gauze and there is always a dilemma that the diagnosis could be anything at all. ${ }^{10}$ It is important for the pathologist to remember that unlike fine needle aspiration procedure at other sites where a separate biopsy procedure is used to sample tissue to clarify doubts arising in cytological evaluation, the cytological assessment here is a component of the biopsy procedure and our impression is a prelude to the final diagnosis on histology. The cytological impression can be built upon after histological analysis, but we should not deviate from the initial commitment on cytology. The level of commitment has to be tailored to individual case.

\section{Sample and information dispatch to the laboratory}

A previous communication with the neurosurgeon should ensure that specimens for immediate cytological evaluation arrive in the laboratory in normal saline when the pathologist is present. The pathologist should discharge the specimen in a $10 \%$ formalin container for histological processing after preparing smears from representative areas of all the pieces. Age of the patient, location of the lesion and the imaging information including the impression and / or the differential diagnosis are the three mandatory components that need to be present in all request forms accompanying the specimens if a sensible impression is to be relayed after

Table 1: Common intra-axial tumours in adults

\begin{tabular}{ll}
\hline Supratentorial & \multicolumn{1}{c}{ Infratentorial } \\
\hline Metastases & Metastases \\
\hline Diffusely infiltrating astrocytomas & Hemangioblastoma \\
\hline Oligodendroglial tumours & \\
\hline Mixed oligoastrocytomas & \\
\hline
\end{tabular}

Table 2: Common intra-axial tumours in children

\begin{tabular}{ll}
\hline Infratentorial & \multicolumn{1}{c}{ Supratentorial } \\
\hline Pilocytic astrocytoma & Pleomorphic xanthoastrocytoma \\
Medulloblastoma & Diffusely infiltrating astrocytomas \\
Ependymoma & Primitive neuroectodermal tumour \\
\hline
\end{tabular}

Table 3: WHO grades of CNS tumours

\begin{tabular}{|c|c|c|c|c|}
\hline Astrocytic tumours & I & II & III & IV \\
\hline Pilocytic astrocytoma & * & & & \\
\hline Diffuse astrocytoma & & $*$ & & \\
\hline Anaplastic astrocytoma & & & $*$ & \\
\hline Glioblastoma & & & & $*$ \\
\hline \multicolumn{5}{|l|}{ Oligodendroglial tumours } \\
\hline Oligodendroglioma & & $*$ & & \\
\hline Anaplastic oligodendroglioma & & & $*$ & \\
\hline \multicolumn{5}{|l|}{ Oligoastrocytic tumours } \\
\hline Oligoastrocytoma & & $*$ & & \\
\hline Anaplastic oligoastrocytoma & & & * & \\
\hline \multicolumn{5}{|l|}{ Ependymal tumours } \\
\hline Ependymoma & & & $*$ & \\
\hline Anaplastic ependymoma & & & & $*$ \\
\hline \multicolumn{5}{|l|}{ Meningeal tumours } \\
\hline Meningioma & * & & & \\
\hline Atypical meningioma & & $*$ & & \\
\hline Anaplastic meningioma & & & * & \\
\hline \multicolumn{5}{|l|}{$\begin{array}{l}\text { Tumours of cranial and paraspinal } \\
\text { nerves }\end{array}$} \\
\hline Schwannoma & * & & & \\
\hline \multicolumn{5}{|l|}{ Embryonal tumours } \\
\hline Medulloblastoma & & & & $*$ \\
\hline \multicolumn{5}{|l|}{ Tumours of sellar region } \\
\hline Craniopharyngioma & $*$ & & & \\
\hline
\end{tabular}


cytological evaluation. ${ }^{9}$ It is always wise to impress upon the management team comprising of the neurosurgeon, radiologist and pathologist. Regular interactive sessions with the management team not only helps one to understand the needs and limitations of the other members of the team, but helps to keep the communication channels open so that genuine concerns can be immediately addressed for optimal patient management.

\section{Preparation of smears, fixation and staining}

Pinhead size of tissue is placed near one end of a slide and the flat surface of another slide is placed on top of the specimen and advanced with a uniform motion without exerting undue pressure to create a crush smear. It helps to remember the ease with which the tissue can be spread into a smear because lot of intercellular collagen or fibres in meningiomas, schwannomas and low-grade astrocytomas cause difficulty in preparing smears while high-grade tumours spread with ease.The smear is fixed immediately for one minute in a fixative containing a mixture of $95 \%$ isopropyl alcohol and 5\% glacial acetic acid.Hematoxylin and eosin staining is completed in 15 minutes by bringing the fixed smears down to water by placing the smear in $80 \%$ and $70 \%$ alcohol for one minute each followed by five dips in water, five minutes in hematoxylin, four dips in water, five dips in $1 \%$ acid alcohol, four dips in water, four dips in $1 \%$ lithium carbonate, four dips in water, ten seconds in $2 \%$ eosin, four dips in water, one minute each in $70 \%, 80 \%$ and absolute alcohol followed by two minutes in xylene before being mounted. Eosin defines the fibrillary background very faithfully for confident evaluation. ${ }^{11}$

\section{Prelude to the evaluation process}

The pathologist can best utilize the 15 minutes it takes for the smears to be prepared and stained by going through a quick mental review of the common causes and location of pediatric and adult tumours. It will help to remember that posterior fossa is the preferred location in children and anterior fossa in adults in two thirds of cases each. The most common tumours in children are pilocytic astrocytoma, ependymoma and medulloblastoma. Astrocytoma of various grades, oligodendroglioma, meningioma and metastatic carcinoma are the most common neoplasms in adults. The common intra-axial tumours in adults and children with respect to their supratentorial or infratentorial locations are reproduced in Table 1 and $2 .{ }^{12}$

A basic understanding of the WHO grading of CNS tumours is necessary because this grading system predicts the biologic behaviour of these tumours most accurately and is highly reproducible among pathologists. Tumours are classified into four grades according to this system. Grade I tumours are characterized by slow growth without a potential for malignant transformation. Cyst with a mural nodule is the usual image for intraparenchymal grade I tumours typified by pilocytic astrocytoma. Being circumscribed lesions these tumours are susceptible to surgical cure. Hypercellularity and cellular atypia in the form of pleomorphism and hyperchromatism are the usual features in these tumours . Mitotic figures are rare and microvascular proliferation and necrosis are not present.

WHO grade II tumours are infiltrative lesions that have a tendency to recur.Malignant transformation with evolution to higher grades is the rule. A rare mitotic figure may be present, but microvascular proliferation and necrosis are not evident.

WHO grade III tumours are characterized by faster growth and shorter survival. Mitotic figures are frequent and microvascular proliferation and/or necrosis are allowed except for purely astrocytic tumours.

WHO grade IV tumours are characterized by either undifferentiated small round cell morphology or the presence of microvascular proliferation and / or necrosis in mitotically active astrocytic tumours.

The common CNS tumours are classified according to the WHO grades in Table $3 .^{13}$

\section{Evaluation process}

After a quick mental appraisal of the common possibilities, the case at hand will be easier to decipher if the evaluation process follows a systematic approach by asking and answering the following questions sequentially. ${ }^{10}$

1. Could this be from a normal tissue?

2. Could this be a process that mimics a neoplasm?

3. Could this be reactive gliosis?

4. Could this be a neoplasm?

5. Could this be a particular neoplasm?

\section{Could this be from a normal tissue?}

Normal cerebellar granular layer cells can produce a very cellular smear comprising of sheets of small cells and can be easily mistaken for medulloblastoma in children and lymphoma and metastatic small cell carcinoma in adults if due attention is not paid to the bland nature of the cells (fig.1). Neurological specimens may not be fully representative of the lesion or may not be in the lesion at all, despite neuroimaging guidance. Even millimeters difference in some lesions can determine whether or not diagnostic material is received by the pathologist. The author once avoided a possible hasty diagnosis of a choroid plexus papilloma by raising its possibility to the neurosurgeon who confidently stated that the normal choroid plexus could have been picked up during stereotactic sampling of a periventricular lesion (fig. 2). 

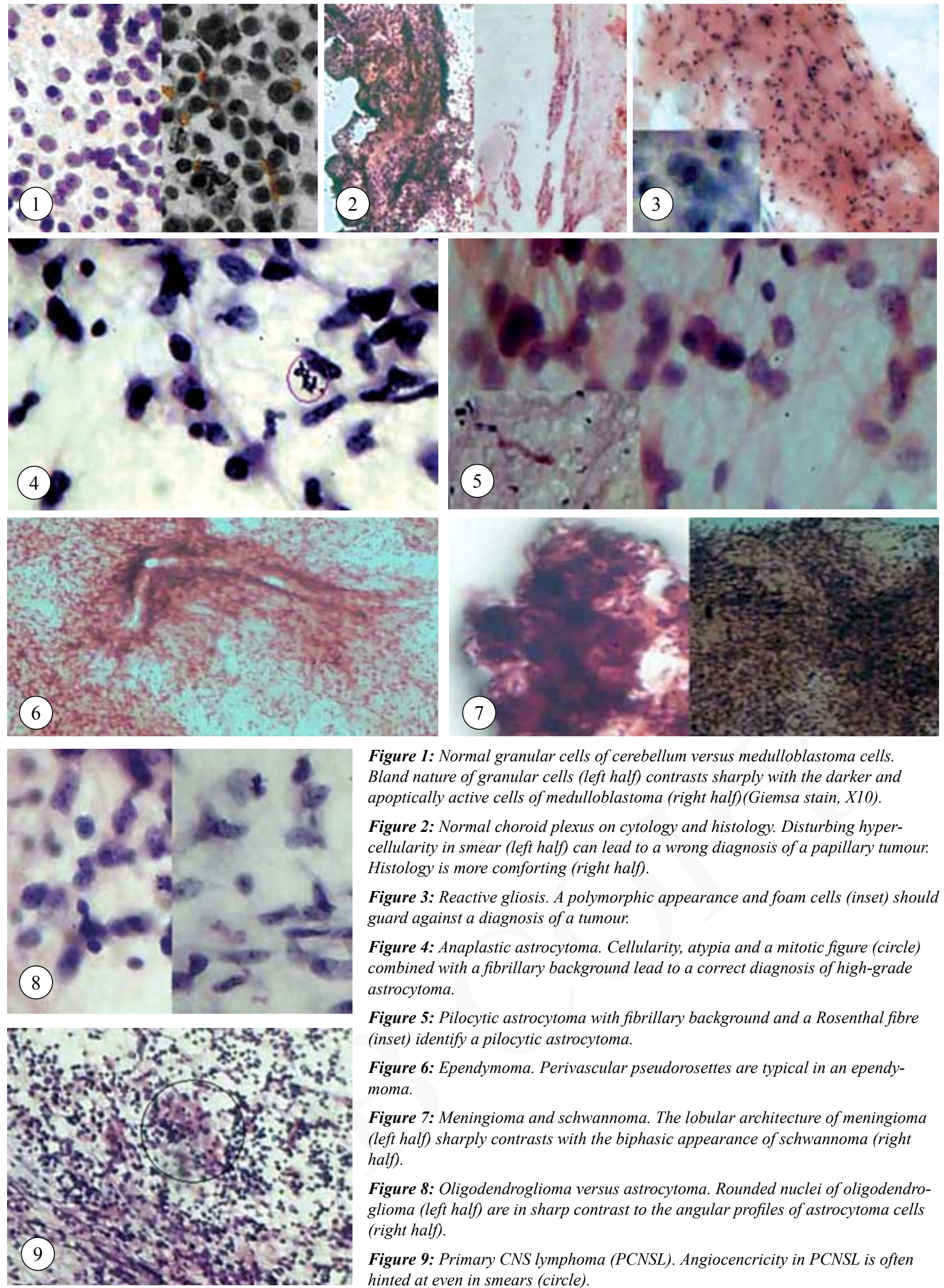

Figure 1: Normal granular cells of cerebellum versus medulloblastoma cells. Bland nature of granular cells (left half) contrasts sharply with the darker and apoptically active cells of medulloblastoma (right half)(Giemsa stain, X10).

Figure 2: Normal choroid plexus on cytology and histology. Disturbing hypercellularity in smear (left half) can lead to a wrong diagnosis of a papillary tumour. Histology is more comforting (right half).

Figure 3: Reactive gliosis. A polymorphic appearance and foam cells (inset) should guard against a diagnosis of a tumour.

Figure 4: Anaplastic astrocytoma. Cellularity, atypia and a mitotic figure (circle) combined with a fibrillary background lead to a correct diagnosis of high-grade astrocytoma.

Figure 5: Pilocytic astrocytoma with fibrillary background and a Rosenthal fibre (inset) identify a pilocytic astrocytoma.

Figure 6: Ependymoma. Perivascular pseudorosettes are typical in an ependymoma.

Figure 7: Meningioma and schwannoma. The lobular architecture of meningioma (left half) sharply contrasts with the biphasic appearance of schwannoma (right half).

Figure 8: Oligodendroglioma versus astrocytoma. Rounded nuclei of oligodendroglioma (left half) are in sharp contrast to the angular profiles of astrocytoma cells (right half).

Figure 9: Primary CNS lymphoma (PCNSL). Angiocencricity in PCNSL is often hinted at even in smears (circle). 
Interaction with the neurosurgeon during intraoperative consultation can often prompt him to do a serial sampling approach that will achieve a proper diagnosis for our mutual patient. Goal of rapid diagnostic procedure is to preserve some tissue that is assured to be lesional and representative. ${ }^{14}$

\section{Could this be a process that mimics a neoplasm?}

The presence of significant proportion of macrophages, neutrophils, lymphocytes, granulomas and abnormal vascular structures should guard one against a hasty diagnosis of a tumour. Infarction should be considered if macrophages are the predominating cells (figure 3).

Abscesses head the list of differential if neutrophils dominate the smears. Tuberculoma is high on the cards if granulomas are noted while recognizing the presence of granulomas in neoplasms like germinomas. Lymphocytes should alert one to the presence of a reactive process or infection while remembering that lymphocytes can be sampled from many neoplasms like pleomorphic xanthoastrocytoma, gemistocytic astrocytoma, dysgerminoma and last but not the least, malignant lymphoma. Eye-catching abnormal vascular profiles should prompt one to consider vascular malformations while carefully ruling out ependymomas, pilocytic astrocytomas, schwannomas and high-grade glioma.

\section{Could this be reactive gliosis?}

Lack of hypercellularity and the presence of a polymorphic composition are two cytological features that should make one think twice before diagnosing a neoplasm (fig. 3). ${ }^{14}$

Reactive lesions do not usually yield a uniformly hypercellular smear and the cellular composition is not monomorphic but polymorphic with inflammatory cells being admixed with glial cells.

\section{Could this be a neoplasm?}

Hypercellularity, cellular atypia in the form of pleomorphism and hyperchromatism and mitotic activity all combine to confidently render a diagnosis of a tumour (fig. 4).

\section{Could this be a particular neoplasm?}

It pays to go through the following questions once an impression of a tumour is formed.

a. Is the background fibrillary or are the tumours elaborating fibres?

b. Are the nonfibrillary cells cohesive or dyshesive and in sheets?

c. Do the cells show features of differentiation?

d. Are the cells small? 5a. Is the background fibrillary or are the tumours elaborating fibres?

Fibrillary background and microvascular proliferation favour a primary neoplasm, but it is always wise to first rule out a lymphoma and metastatic carcinoma in an adult.The entities to consider in children are pilocytic astrocytoma and ependymoma. Rosenthal fibres favour the diagnosis of pilocytic astrocytoma (fig. 5) and perivascular pseudorosettes tips the balance in favour of ependymomas (fig.6). It pays to remember that the looser areas of medulloblastoma also appear fibrillary with tumour cells elaborating fibres. Various grades of diffusely infiltrating astrocytomas are the most common fibrillary tumours in adults. Mitotic figures are a very reproducible criterion and one can confidently render a diagnosis of high-grade glioma if they are frequent in a fibrillary tumour in an adult (fig. 4).

\section{5b. Are the nonfibrillary cells cohesive or dyshesive and in sheets?}

Cohesive clusters in an adult favour a diagnosis of metastasis or meningioma. The obviously malignant cells of a metastatic carcinoma are in sharp contrast to the bland appearing benign glial component if present. The cells of meningioma appear bland and the typical whorls are usually seen popping from the edges of the clusters. The lobular appearance of meningioma differentiates it from schwannoma which has a typical biphasic appearance (fig. 7). Dyscohesive clusters or sheets are a pointer towards oligodendroglioma, pituitary adenoma or malignant lymphoma. The round nuclear profiles of oligodendroglioma contrast sharply with the angular outlines of astrocytoma nuclei (fig. 8).

\section{5c. Do the cells show features of differentiation?}

Glandular or squamous differentiation among malignant cells will obviously lead to a diagnosis of metastatic adenocarcinoma or squamous cell carcinoma respectively.

\section{5d. Are the cells small?}

Small apoptically or mitotically active cells in a child favours a medulloblastoma (fig.1). Lymphoma and metastatic small cell carcinoma are the major differential in adults. The nuclear moulding of small cell carcinoma is absent in lymphoma where the cells are dispersed. Primary CNS lymphoma often demonstrates the typical angiocentric and angioinvasive patterns in histology and this diagnostic feature may be hinted at in cytological smears (fig. 9). Romanowsky stain highlights the lymphoglandular bodies in the background of lymphoma cells and may come handy in difficult cases to identify a small cell neoplasm as lymphoma. Oligodendroglioma nuclei can also appear uniformly small more often than not and should always be considered in the differential of small cells. Pituitary 
adenoma cells are also small, but they are more uniform and monomorphic than lymphoma or metastatic small cell carcinoma.

\section{Reporting on smears}

After the analysis is complete, the final reporting on smears usually follows one of the following three options:

1. Definite diagnosis can be issued if cytological evaluation results in a conclusion that matches the intraoperative and imaging impressions or confidently addresses the diagnostic dilemma. An abscess can be reliably separated from a glioblastoma or a metastasis during evaluation of a ring enhancing lesion.

2. Definite diagnosis cannot be issued because cytological conclusion differs from intraoperative and imaging impressions. A hint to the grade of the tumour, either low-grade (WHO Grades I and II) or high-grade (WHO Grades III and IV), combined with fibrillary or nonfibrillary nature of the tumour can be issued with a note to correlate the cytological impression with the final histological diagnosis.

3. No clue to the diagnosis can be suggested and has to be accompanied with a note to await histopathological report for fuller and complete evaluation.

\section{CONCLUSION}

Intraoperative smears of neurosurgical specimens permit rapid and accurate diagnosis if a systematic approach is implemented in the analytical process. Cytological evaluation should always be followed by a histological confirmation whenever possible. Age of the patient, location of the lesion and the imaging impression are the three mandatory components of the request form accompanying the specimen. Cytological report on intraoperative smears should both provide a preliminary diagnosis and assure the surgeon that representative lesional tissue has been obtained for definite histological diagnosis. Regular interactive sessions with the management team comprising of the neurosurgeon, radiologist and pathologist help to reinforce in all the truth that cytological diagnosis can never be the sole prerogative of the pathologist and can only be as reliable as the combined intelligence of the three specialists.

\section{ACKNOWLEDGEMENT}

I wish to express deep regards to Prof. UP Devkota, Neurosurgeon and Dr. Ram Kumar Ghimire, Consultant radiologist for their support to prepare this article.

\section{REFERENCES}

1. Chandana SR, Movva S, Arora $\mathrm{M}$ et al. Primary brain tumours in adults. Am Fam Physician 2008;77:1423-30.

2. Rosemberg S, Fujiwara D. Epidemiology of pediatric tumors of the nervous system according to the WHO 2000 classification: A report of 1195 cases from a single institution. Childs Nerv Syst 2005;21:940-4.

3. Jemal A, Siegel R, Ward E et al. Cancer statistics, 2006. J Clin 2006; 56:106-30.

4. Savargaonkar P, Farmer PM. Utility of intra-operative consultations for the diagnosis of central nervous system lesions. Ann Clin Lab Sci 2001;31:133-9.

5. Kini JR, Jeyraj V, Jayaprakash CS et al. Intraoperative consultation and smear cytology in the diagnosis of brain tumours. KUMJ 2008; 6: 453-7.

6. Roessler K, Dietrich W, Kitz K. High diagnostic accuracy of cytologic smears of central nervous tumors: A 15-year experience based on 4,172 patients. Acta Cytol 2002;46:667-74.

7. Plesec TP, Prayson RA. Frozen section discrepancy in the evaluation of central nervous system tumours. Arch Pathol Lab Med 2007; 131: $1532-40$.

8. Hall WA, Liu H, Martin AH et al. Comparison of stereotactic brain bipsy to interventional magnetic-resonance-imaging fuided brain biopsy. Stereotact Funct Neurosurg 1999;73:148-53.

9. Prayson RA, Cohen ML. Intraoperative consultation. In: Practical differential diagnosis in surgical neuropathology. Totowa, New Jersey: Humana Press Inc., 2000: pp1-3.

10. Kleinschmidt-DeMasters BK, Prayson RA. An algorithmic approach to the brain biopsy. Arch Pathol Lab Med 2006;130:1630-48.

11. Timperley WR. Cerebrospinal fluid examination and direct brain preparations. In: Gray W, McKee GT, eds. Diagnostic cytopathology. China: Churchill Livingstone, 2003: pp954-6.

12. Smithuis R, Montanera W. Brain tumour - systematic approach. [homepage on the Internet]. Toronto, Canada: 20080702.(Cited 15 June 2011). Available from: http://www.radiologyassistant.nl/ en/47f86aa182b3a

13. Kleihues P, Louis DN, Wiestler OD et al. WHO grading of tumours of the central nervous system. In: Louis DN, Ohgaki H, Wiestler OD et al, eds. WHO classification of tumours of the central nervous system. Lyon: IARC, 2007.pp10-11.

14. Slowinski J, Harabin-Slowinska M, Mrowka R. Smear technique in the intra-operative brain tumour diagnosis: Its advantages and limitations. Neurol Res 1999;21:121-4. 